\title{
Invariant curves and semiconjugacies of rational functions
}

\author{
Alexandre Eremenko* \\ October 15, 2018
}

\begin{abstract}
Jordan analytic curves which are invariant under rational functions are studied.

Keywords: rational functions, iteration, functional equations.

MSC 30D05, 37F10.
\end{abstract}

In the last paragraph of his memoir [6] on iteration of rational functions Fatou writes:

"Il nous resterait à étudier les courbes analytiques invariantes par une transformation rationnelle et dont l'étude est intimement liée á celle des fonctions étudiées dans ce Chapitre. Nous espérons y revenir bientôt 1 1

As far as I know, Fatou never returned to this question in his published work. Neither I know of any systematic study of the question after Fatou. If a Jordan analytic invariant curve is the boundary of a domain of attraction, then it must be a circle, [6].

Which Jordan analytic curves $\gamma$ in the Riemann sphere can be invariant under rational functions? Of course, $\gamma$ can be a circle, and it is easy to describe all rational functions which leave a given circle invariant: such functions must commute with the reflection in this circle.

\footnotetext{
*Supported by NSF grant DMS-1067886.

${ }^{1}$ It remains to study analytic curves invariant under rational transformations, which are intimately connected with the functions studied in this chapter. We hope to return to this soon.
} 
Other examples are obtained as level lines of the linearizing functions in Siegel discs or Hermann rings. It is not known whether a Jordan analytic invariant curve, different from a circle, and which is mapped onto itself homeomorphically can intersect the Julia set.

If $f$ is a polynomial (or an entire function) then the only possible Jordan invariant analytic curves in $\mathbf{C}$ are either circles or preimages of circles under linearizing functions of Siegel discs [1].

Przytycki asked whether a rational function $f$ can have a repeller, which is a Jordan analytic curve different from a circle. A repeller $\gamma$ is a compact set which has a neighborhood $V$, such that for $z \in V, f^{n}(z) \in V$ if and only if $z \in \gamma$.

The principal result of this paper implies that such Jordan analytic repellers must be algebraic curves, except for the Lattés examples. However, the only example of a rational function having such a repeller, different from a circle, that I could produce, is a Lattés example.

Theorem 1. Let $f$ be a rational function and $\gamma$ a Jordan analytic invariant curve such that $\left.f\right|_{\gamma}$ is not a homeomorphism and there is a repelling periodic point $a \in \gamma$ with multiplier $\lambda,|\lambda|>1$. Assume in addition one of the following:

a) $\gamma$ does not contain critical or neutral rational fixed points of $f$, or

b) $\gamma \subset J(f)$.

Then $\lambda$ is real, and there exist a non-hyperbolic Riemann surface $S$ with an anti-conformal involution $s$ and an endomorphism $g: S \rightarrow S$, and a holomorphic map $h: S \rightarrow \overline{\mathbf{C}}$ such that $g \circ s=s \circ g, h(X) \subset \gamma$ where $X \subset S$ is the set of points fixed by the involution s, and the following semi-conjugacy relation holds:

$$
h \circ g=f^{n} \circ h
$$

with some integer $n \geq 0$.

Corollary. Assumptions of Theorem 1, imply that the curve $\gamma$ is algebraic, or $f$ is a Lattés example.

If $\gamma$ is a repeller, then all assumptions of Theorem 1, including both a) and $\mathrm{b}$ ) are satisfied.

Proof of Theorem 1. Replacing $f$ by some iterate, we reduce to the case that the point $a$ is fixed. Let $F$ be the Poincaré function associated with the 
fixed point $a$. This means that

$$
F(\lambda z)=f \circ F(z), \quad F(0)=a, \quad F^{\prime}(0) \neq 0 .
$$

Such function exists for every repelling fixed point and it is meromorphic in the plane $\mathbf{C}$. Let $\Gamma$ be the component of $F^{-1}(\gamma)$ that contains 0 . Then the intersection of $\Gamma$ with a neighborhood of 0 is a smooth curve invariant under the map $z \mapsto \lambda^{-1} z$, which implies that $\lambda$ is real. Replacing $f$ by the second iterate we achieve that $\lambda>1$. Then it follows that $\Gamma$ contains a straight line, and without loss of generality we may assume that this is the real line. We have $F(\mathbf{R}) \subset \gamma$.

Consider the restriction mappings $\mathbf{R} \rightarrow \gamma$ of all Poincaré maps $F_{j}$ for all repelling fixed points $a_{j}$ on $\gamma$. There are finitely many of them, as the number of fixed points is finite. We claim that under our assumptions at least one of these maps is not injective.

Indeed, suppose that some $F_{j}$ is injective on the real line. Then the image $F_{j}(\mathbf{R})$ is a simple arc $\gamma_{j} \subset \gamma$, and it is easy to see that the endpoints of this arc must be attracting fixed points with real multipliers.

Indeed these endpoints are fixed because $\gamma_{j}$ is invariant and the iterates of $f$ converge to these endpoints. This convergence implies that these endpoints cannot be repelling.

Suppose that the assumption b) in Theorem 1 holds. As $\gamma_{j}$ is a subset of a Jordan analytic curve, its endpoints cannot be neutral irrational points. A local description of dynamics near a neutral rational fixed point shows that if such a point $w$ is an endpoint of a smooth invariant curve on which the iterates converge to $w$, then this curve must intersect the set of normality. This contradiction completes the proof of non-injectivity of $\left.F_{j}\right|_{\mathbf{R}}$ under the assumption b).

We continue the non-injectivity proof under the assumption a). The endpoints of $\gamma_{j}$ can coincide in which case we obtain that $\left.f\right|_{\gamma}$ is a homeomorphism. Now let $b$ be an endpoint of $\gamma_{j}$ which does not coincide with the other endpoint. Assumption a) implies that the endpoints are attracting. From the local description of the dynamics near an attracting fixed point $b$ we conclude that there is a simple invariant $\operatorname{arc} \delta_{0}$ disjoint from $\gamma_{j}$ such that $\gamma_{j} \cup \delta_{0} \cup\{b\}$ is an arc of $\gamma$. For $k \geq 1$, let $\delta_{k}$ be the component of $f^{-1}\left(\delta_{k-1}\right)$ which contains $\delta_{k-1}$. Then

$$
\Delta=\cup_{k=1}^{\infty} \delta_{k}
$$

is a simple invariant curve, $\Delta \subset \gamma$, one endpoint of $\Delta$ is $b$ and the other endpoint is a repelling fixed point $a_{k}$ on $\gamma$ distinct from $a_{j}$. This means 
that the curves $\gamma_{j}$ and $\gamma_{k}$ have a common endpoint $b$, and their union is an analytic curve near this common point.

We conclude that our curves $\gamma_{j}$ cover $\gamma$, and $\left.f\right|_{\gamma}$ is a homeomorphism. This contradicts the assumptions and proves the claim.

From now on we assume that some Poincaré function $F$ has non-injective restriction on the real line.

We recall a result of [4]. Let $F: \mathbf{C} \rightarrow S$ be a non-constant holomorphic map from the complex plane to a Riemann surface $S$. Consider the following equivalence relation in $\mathbf{C}: x \sim y$ iff $F(x)=F(y)$. Let $\Gamma_{F} \subset \mathbf{C}^{2}$ be the graph of this equivalence relation. It is easy to see that $\Gamma_{F}$ is an analytic variety of pure (complex) dimension 1 (has no isolated points).

Now suppose that a analytic variety $\Gamma \subset \mathbf{C}^{2}$ of pure dimension 1 which is a graph of an equivalence relation is given. Then there exists a holomorphic map $F: \mathbf{C} \rightarrow S$, where $S$ is a non-hyperbolic Riemann surface such that $F(x)=F(y)$ iff $(x, y) \in \Gamma$. This map $F$ is defined by $\Gamma$ uniquely up to a composition with an automorphism of $S$.

Now we characterize the graphs $\Gamma$ of equivalence relations corresponding to Poincaré functions.

Lemma 1. A holomorphic map $F: \mathbf{C} \rightarrow S$ to a non-hyperbolic Riemann surface $S$ is a Poincaré function of an endomorphism of $S$ if and only if $\Gamma_{F}$ is invariant under a map

$$
(x, y) \mapsto(\lambda x, \lambda y)
$$

for some $\lambda \in \mathbf{C},|\lambda|>1$.

Proof. That the graph $\Gamma_{F}$ corresponding to a function $F$ satisfying (2) is invariant with respect to this map is clear.

To prove the converse statement, we recall the result from [4] that the existence of a decomposition $F=f \circ G$, where $f$ and $G$ are maps between non-hyperbolic Riemann surfaces is equivalent to the inclusion $\Gamma_{G} \subset \Gamma_{F}$. Let $\Gamma_{1}=\lambda \Gamma_{F} \subset \Gamma_{F}$. Then $\Gamma_{1}=\Gamma_{G}$ where $G(\lambda z)=F(z)$. On the other hand, by the result of [4] just cited, $F=f \circ G$ where $f: S \rightarrow S$ is an endomorphism. So $F(\lambda z)=f \circ G(\lambda z)=f \circ F(z)$, that is $F$ satisfies a Poincaré equation. Putting $z=0$ we obtain that $F(0)$ is a fixed point of $f$, and the multiplier of this fixed point is $\lambda$ by the chain rule.

This completes the proof of the lemma. 
A holomorphic map $F: \mathbf{C} \mapsto S$ will be called real if there exists an anti-conformal involution $s: S \rightarrow S$ such that $F(\bar{z})=s \circ F(z)$.

Lemma 2. A holomorphic map $F: \mathbf{C} \rightarrow S$ is real if and only if $\Gamma_{F}$ is invariant under the map

$$
(x, y) \mapsto(\bar{x}, \bar{y}) .
$$

This is clear.

Now we return to the proof of Theorem 1 . As $F$ is not injective on the real line, there is a real analytic germ $\phi \neq$ id such that $F \circ \phi=F$. This implies that some part $\Gamma_{1} \neq\{(x, x): x \in \mathbf{C}\}$ of the graph $\Gamma_{F}$ is parametrized by $(x, \phi(x))$, so this part is invariant under the map (44).

Now let $\Gamma_{2} \subset \Gamma_{F}$ be the smallest analytic variety of pure dimension 1 which contains $\Gamma_{1}$, which is a graph of an equivalence relation and which is invariant under the maps (44) and (3).

Proof of existence of $\Gamma_{2}$. Let $E \subset \mathbf{C}^{2}$ be an arbitrary set containing the diagonal $D=\{(x, x): x \in \mathbf{C}\}$. Consider three operations on such sets:

$$
\begin{gathered}
E \mapsto \Lambda E=\left\{\lambda^{n}(x, y):(x, y) \in E, n \in \mathbf{N}\right\}, \\
E \mapsto S E=\{(x, y):(y, x) \in E\}, \\
E \mapsto T E=\{(x, z): \exists y,(x, y) \in E,(y, z) \in E\} .
\end{gathered}
$$

If $E$ does not contain isolated points then $\Lambda E, S E, T E$ do not contain isolated points. Moreover, if $E$ is symmetric with respect to the operation (44) then each $\Lambda E, E S, T E$ is also symmetric with respect to this operation. Now we apply all finite sequences of operations $\Lambda, S, T$ to to $\Gamma_{1} \cup D$, and take as $\Gamma_{2}$ the union of those irreducible components of $\Gamma_{F}$ that have non-isolated intersection with the resulting sets. Then $\Gamma_{2}$ is the minimal analytic set of pure dimension 1, which is a graph of an equivalence relation, invariant with respect to (3), and by the previous remark, it is symmetric with respect to (44). This completes the construction of the set $\Gamma_{2}$.

If $\Gamma_{2}=\Gamma_{F}$ then $F$ is real and $\gamma$ is a circle. If $\Gamma_{2} \neq \Gamma_{F}$ then there exists a factorization

$$
F=h \circ G,
$$

where $G$ is a Poincaré function of some endomorphism $g: S \rightarrow S$ of a non-hyperbolic surface $S$, that is

$$
G(\lambda z)=g \circ G(z),
$$


$\Gamma_{2}=\Gamma_{G}$, and $h: S \rightarrow \overline{\mathbf{C}}$ is a holomorphic map. Moreover, $G$ is real. Combining (5) and (6) we obtain

$$
f \circ h \circ G(z)=f \circ F(z)=F(\lambda z)=h \circ G(\lambda z)=h \circ g \circ G(z),
$$

and this implies (11). This completes the proof of Theorem 1.

To prove the Corollary, we discuss the functional equation (1). First of all, $S$ can be a torus, $\mathbf{C}^{*}, \mathbf{C}$ or $\overline{\mathbf{C}}$. If $S$ is a torus, then $f$ is a Lattés example. If $S$ is $\mathbf{C}$ or $\mathbf{C}^{*}$, and $h$ has an essential singularity then $f$ also must be a Lattés example. This was proved in [3]. See also [2] for another proof. Otherwise $S=\overline{\mathbf{C}}$, and thus both $g$ and $h$ are rational. This proves the corollary.

Lattés examples indeed have Jordan analytic invariant curves which are not circles. These curves can be algebraic or transcendental.

Example 1. Let $\wp$ be the Weierstrass function with periods $2 \omega_{1}$ and $2 i \omega_{2}$ where we assume that $\omega_{1}$ and $\omega_{2}$ are real. Consider the line $L=\left\{x+2 i \omega_{2} / 3\right.$ : $x \in \mathbf{R}\}$ and let $\gamma=\wp(L)$. The simplest Lattés function $f$ corresponding to $\wp$ satisfies

$$
\wp(2 z)=f \circ \wp(z)
$$

and we see that $\gamma$ is invariant under $f$, because $2 L \equiv-L$ modulo periods, and $\wp$ is even. It is easy to see that $\gamma$ is a Jordan analytic curve which is not a circle, and $f: \gamma \rightarrow \gamma$ is a two-sheeted covering map.

Let us show that $\gamma$ is algebraic. Let $s(z)$ be the reflection in the line $L$. Define

$$
X(z)=(\wp(z)+\overline{\wp(s(z))}) / 2
$$

and

$$
Y(z)=(\wp(z)-\overline{\wp(s(z))}) /(2 i)
$$

Then $X$ and $Y$ are elliptic functions with the same period lattice as $\wp$. So they are related by an algebraic equation

$$
F(X, Y)=0
$$

When $z \in L$, we have $s(z)=z$, so $X(z)=\operatorname{Re} \wp(z)$ and $Y(z)=\operatorname{Im} \wp(z)$. So points $x+i y$ on our curve $\gamma=\wp(L)$ satisfy the algebraic equation $F(x, y)=0$.

Example 2. Let $\wp$ be the Weierstrass function with primitive periods $(1, \tau)$, where $\tau=p+i$, where $p$ is real and irrational. Let $\gamma=\wp(L)$, where $L$ is the 
same as Example 1 above, and $f$ is a Lattés function as in (7). It is easy to see that $\gamma$ is a Jordan analytic curve invariant under $f$, which is not a circle.

Let us show that $\gamma$ is transcendental. Consider the function $g(z)=$ $\overline{\wp(s(z))}$, where $s$ is the reflection with respect to $L$. It is an elliptic function with periods 1 and $\bar{\tau}=p-i$. Evidently, the period $p-i$ is not a rational combination of 1 and $p+i$. So $g$ is an elliptic function whose lattice is not commensurable with the lattice of $\wp$. Suppose now that $\gamma$ is algebraic and let $F(x, y)=0$ be the equation of $\gamma$. Then

$$
F((\wp+g) / 2,(\wp-g) /(2 i))=0
$$

holds on the real line and thus everywhere. We conclude that $\wp$ and $g$ are algebraically dependent, and this is a contradiction, because algebraically dependent elliptic functions must have commensurable lattices. This proves that $\gamma$ is transcendental.

Equation (11) in rational functions was recently studied in [8] and [11]. It is closely related to factorization theory of rational functions with respect to composition which is due to J. Ritt [12, 14, 13]. Pakovich [11] obtained a complete classification of triples of rational functions that satisfy (1). However, it is hard to obtain from this classification a description of triples that satisfy other conditions of Theorem 1 .

The simplest solutions of (1) with $n=1$ can be constructed as follows [8]:

Let $u$ and $v$ be two rational functions. Set

$$
f=u \circ v, \quad g=v \circ u \text { and } h=u .
$$

Then $h \circ g=f \circ h$, so (1) is satisfied.

Another class of examples is obtained by taking an arbitrary rational function $w$ and setting $f(z)=z^{m} w^{n}(z) g(z)=z^{m} w\left(z^{n}\right)$ and $h(z)=z^{n}$.

These examples do not exhaust all possibilities which are listed in [11.

It is interesting to know whether (8) leads to Jordan analytic invariant curves which are not circles. Such examples will occur if

$$
v \circ u
$$

is a real rational function but $u$ is not real and maps the circle $\mathbf{R} \cup\{\infty\}$ onto a Jordan analytic curve $\gamma$ which is not a circle. Then $\gamma$ will be an invariant curve for $u \circ v$. 
Example 3. (F. Pakovich). Let $P_{n}(z)=z^{n}$ and $J(z)=\left(z+z^{-1}\right) / 2$. It is well known that

$$
R:=J \circ P_{n}=T_{n} \circ J,
$$

where $T_{n}$ are real polynomials (they are Chebyshev polynomials normalized so that the leading coefficient is $\left.2^{n-1}\right)$. Now, if $\epsilon=\exp (2 \pi i / n)$, then the first factorization implies that $R(\epsilon z)=R(z)$. So $R(z)=T_{n} \circ J(\epsilon z)$. Let

$u(z)=J(\epsilon z)$. Then $u(\mathbf{R})$ is a hyperbola $\gamma$, it is mapped by $T_{n}$ on the real line, and this hyperbola is invariant under the map $f=u \circ T_{n}$.

However a hyperbola, when considered on the Riemann sphere, is not a Jordan curve. Thus the only examples we have to illustrate Theorem 1 are Examples 1 and 22 .

The semiconjugacy equation (1) for rational functions occurs also in [9] and [7] in different contexts.

The author thanks the referee for valuable remarks.

\section{References}

[1] Y. Azarina, Invariant analytic curves for entire functions, Siberian Math. J. 30 (1989) 349-353.

[2] X. Buff and A. Epstein, From local to global analytic conjugacies, Ergodic Theory Dynam. Syst. 27 (2008) 1074-1094.

[3] A. Eremenko, Some functional equations connected with iteration of rational functions, Leningrad Math. J., 1, 4 (1990), 905-919.

[4] A. Eremenko and L. Rubel, Arithmetic of entire functions under composition, Adv. Math.,124, 2 (1996), 334-354.

[5] Alexandre Eremenko (mathoverflow.net/users/25510), Circles and rational functions, http://mathoverflow.net/questions/103949.

\footnotetext{
${ }^{2}$ After this paper was accepted, Peter Müller [5] found examples of rational functions $f_{1}$ and $f_{2}$, such that $f_{1} \circ f_{2}$ is real but $\gamma=f_{2}(\mathbf{R})$ is a Jordan analytic curve which is not a circle. This curve $\gamma$ is invariant under $f_{2} \circ f_{1}$, thus giving a non-trivial illustration to Theorem 1, and an example of a Jordan analytic repeller. His functions $f_{1}$ and $f_{2}$ come from isogenies of elliptic curves. Actually in Müller's example, both $f_{1} \circ f_{2}$ and $f_{2} \circ f_{1}$ are Lattés maps, but one can consider an arbitrary real rational function $p$ and set $f_{3}=p \circ f_{1}$. Then $g=f_{3} \circ f_{2}, f=f_{2} \circ f_{2}$ and $h=f_{2}$ will satisfy (1) and none of them will be a Lattés map.
} 
[6] P. Fatou, Sur les équations fonctionnelles, Troisième Mémoire, Bull. Soc. Math. France, 48 (1920) 208-314.

[7] R. Grigorchuk and A. Zuk, Ihara zeta function of infinite groups, the KNS spectral measure and integrable maps, Random walks and geometry, 141180. Walter de Gruyter, Berlin, 2004.

[8] H. Inou, Extending local analytic conjugacies, Trans. Amer. Math. Soc., 363 (2011) 331-343.

[9] M. Dabija and M. Jonsson, Endomorphisms of the plane preserving a pencil of curves, Internat. J. math., 19 (2008) 217-221.

[10] F. Pakovich, Prime and composite Laurent polynomials, Bull. Sci. Math. 133 (2009), no. 7, 693-732.

[11] F. Pakovich, On semiconjugate rational functions, arXiv:1108.1900.

[12] J. Ritt, Prime and composite polynomials, Trans. AMS 23 (1922) 51-66.

[13] J. Ritt, Permutable rational functions, Trans. AMS, 25 (1923) 399-448.

[14] J. Ritt, Equivalent rational substitutions, Trans AMS 26 (1924) 221229 .

Department of mathematics, Purdue University, West Lafayette, IN 27907 